Chronic Obstructive Pulmonary Diseases: Journal of the COPD Foundation

\title{
COPD9usa Session Summary \\ Back to the Future: Past, Present, and Future is COPD360
}

\author{
Michael W. Hess, BS, RRT, RCP ${ }^{1}$ and Barry Make, $\mathrm{MD}^{2}$
}

This article serves as a CME-available enduring material summary of the following COPD9uSA presentations:

- "The Past and Present Evidence" presenter: Stephen Rennard, MD

- "COPD: The Way Forward” presenter: Gerard Turino, MD

- “E-Cigarettes-Hope, Hype or Danger?” presenter: Russell Bowler, MD, PhD

- "Looking Forward" presenter: Prescott Woodruff, MD

- "The Future is Now: A Patient Perspective" presenter: Jim Nelson

• “COPD360 \& The Patient-Powered Research Network” presenter: John Walsh

\section{Abstract}

The treatment and management of chronic obstructive pulmonary disease (COPD) have gone through significant changes over the past decade. This plenary session, part of the 2015 COPD9USA conference, examines how the diagnosis and management of COPD has become more individualized as new phenotypes have been identified. In addition, new diagnostic and management tools are evaluated and the importance of including the patient perspective when designing a treatment plan is discussed. Finally, the future integration of technology in research, clinical communication, and disease education is introduced, including how the COPD Foundation is encouraging and facilitating these efforts.

\begin{abstract}
Abbreviations: chronic obstructive pulmonary disease, COPD; respiratory care practitioner, RCP; Global initiative for chronic Obstructive Lung Disease, GOLD; alpha-1 antitrypsin, AAT; COPD Patient-Powered Research Network, COPD PPRN

Citation: Hess MW, Make B. Back to the future: past, present and future is COPD360. Chronic Obstr Pulm Dis(Miami). 2016; 3(1):473478. doi: http://dx.doi.org/10.15326/jcopdf.3.1.2015.0173
\end{abstract}

1 Battle Creek Veterans Administration Medical Center, Battle Creek, Michigan

2 National Jewish Health, Denver, Colorado

\section{Address correspondence to:}

Michael Hess, RRT

Email: mhessrrt@gmail.com

\section{Keywords:}

chronic obstructive pulmonary disease; COPD; emphysema; social media; alpha- 1 antitrypsin deficiency; Patient-Powered Research Network; PPRN;COPD360social

\section{Introduction}

Wisdom attributed to Confucius tells us that in order to define the future, we must study the past. Both study and definition were central components to the second plenary session of COPD9USA, "Back to the Future: Past,
Present, and Future is COPD360.” While the title may initially seem somewhat ungainly, the session lived up to its ideal of a comprehensive, 360-degree view of the current world of chronic obstructive pulmonary disease (COPD) by offering a unique collection of thought leaders in COPD. Each presenter offered a distinct insight on a particular segment of COPD research and management, including reviews of past efforts, current concepts and plans for future development. This holistic approach gave attendees a broad understanding of the medical science of COPD, encouraging clinicians and patients to collaborate and shape the future.

\section{The Past and Present Evidence}

Dr. Stephen Rennard, of the University of Nebraska Medical Center, a frequent contributor to COPD research initiatives and an internationally recognized expert on 
chronic lung disease, reviewed the current knowledge base of COPD research. Dr. Rennard summarized 3 major areas of COPD study: 1) defining the disease state, 2) analyzing the progression of the disease, and 3) appropriate treatment goals.

The diagnosis of COPD has traditionally been tied to a single physiologic test (spirometry), although current research shows that the disease state we call COPD is far more heterogeneous than originally believed. COPD even affects organ systems outside the respiratory tract to varying degrees in patients with differing COPD phenotypes. In light of this knowledge, clinicians will likely require more comprehensive classification schema in order to more appropriately diagnose and stage COPD in the future.

Current research also shows that what Dr. Rennard describes as the natural history of COPD, or the path a patient takes from initial symptoms to diagnosis and beyond, can be as heterogeneous as the disease itself. Referencing Fletcher and Peto's seminal 1977 lung function deterioration curve, ${ }^{1}$ Dr. Rennard described a more diverse set of natural histories, ranging from achieving a lower maximal lung function early in life leading to lower subsequent lung function to normal development followed by a course of exacerbations to the physiologically-normal age-related decline in lung function followed by a period of marked and accelerated decline. Dr. Rennard posited that some cases of COPD may be induced not by lung damage, but by curtailed lung development in youth and adolescence, followed by age-related decline. This remarkable variation in courses may impede research by offering confounding results when studies do not or cannot account for it.

Concluding his presentation with a review of therapeutic goals, Dr. Rennard alluded to the Global initiative for chronic Obstructive Lung Disease (GOLD) goals of reducing both health risks (such as exacerbations and disease progression) and symptom intensity. ${ }^{2}$ He then reminded attendees that the ultimate goal of treatment should not be merely management, but an actual cure. While current treatment regimens focus on disease management and symptom abatement, Dr. Rennard points out that a cure for COPD is entirely within the realm of possibility, referencing Massaro's 1997 transretinoic acid experimentation using rat lung tissue. ${ }^{3}$ While he pointed out that the tissue regeneration Massaro was able to accomplish in rats has not been duplicated in humans, Dr. Rennard asserted this is due to having not yet found the proper techniques rather than physiological impossibility.

\section{COPD: The Way Forward}

Dr. Gerard Turino built on Dr. Rennard's presentation by discussing the mechanisms behind the development of emphysema, including new data from a research project involving alpha-1 anti-trypsin (AAT) deficiency.

Dr. Turino began with an overview of COPD pathogenesis, including the role of inflammatory mediators and the likelihood of an autoimmune influence on the destruction of the elastin matrix in lung tissue. Both this elastin loss and the accumulation of immune-response cells in the lung can be measured and used as clinical indicators of emphysema. In addition, by analyzing and understanding the origin of antigens found in the lung, it may be possible to determine what enzymes are actively producing those antigens, opening the door to targeted chemical treatments to prevent additional lung tissue degradation. Accordingly, Dr. Turino called for additional study of these chemical processes, in order to deepen current understanding of the biochemical mechanisms involved in COPD.

The Randomized, Placebo-controlled Trial of Augmentation Therapy in Alpha-1 Proteinase Inhibitor Deficiency (RAPID) along with its associated extension, demonstrated a significant reduction in the loss of lung tissue density in alpha- 1 patients receiving supplementation using the pharmaceutical Zemaira (alpha-1 proteinase inhibitor [human]). ${ }^{4}$ In addition, the levels of 2 biomarkers of elastic tissue degredation-desmosine and isodesmosine--were measured in the study cohort. Patients receiving supplementation had significantly lower levels of these biomarkers, indicating that the use of proteinase inhibitor reduced the loss of lung tissue in those patients. This benefit was also realized by patients initially receiving sham therapy with the placebo, once they were converted to supplementation with Zemaira during the trial extension.

Dr. Turino summarized the significance of this research by indicating that due to the correlation of serum desmosine and isodesmosine levels to lung density decline as indicated by computed tomography scan, clinicians now have a clinically-relevant biomarker to track the progression of emphysemic lung damage in clinical trials. In addition, if further research were to identify agents that prevented or reversed elastin degradation, those agents could be deployed to slow or reverse the progression of some forms of COPD. 


\section{E-Cigarettes: Hope, Hype or Danger?}

Switching from disease management to disease progression, Dr. Russell Bowler of National Jewish Health reviewed electronic cigarettes. Considered by some to be potentially of use as a smoking cessation strategy, electronic cigarettes are a controversial topic for many in the COPD world, and thus represent part of the present of COPD research. After presenting statistics showing a marked and concerning increase in electronic cigarette use from 2013 to 2015, especially in middle- and high-school students (Figure 1), Dr. Bowler presented some of the hope for electronic cigarettes in the form of 2 studies intended to demonstrate abstinence from tobacco cigarettes after the addition of their electronic counterparts. ${ }^{5,6}$ However, neither study was able to conclusively determine the effectiveness of electronic cigarettes for this application, especially as compared to existing tobacco cessation strategies, such as nicotine patches. What both studies did demonstrate was the unfortunate realities of any smoking cessation program, which includes poor compliance with therapy, high relapse rates at 6 months post-intervention, and a low rate of utilization of counseling, a key component to successful tobacco cessation.

Dr. Bowler pointed out that both studies appeared to indicate that while the use of electronic cigarettes may not be associated with enhanced abstinence rates, they do appear to be associated with some reduction in the overall number of tobacco cigarettes used. However, this is also not necessarily good news. According to a third study presented by Dr. Bowler, the same toxins present in

\section{Figure 1. Hope, Hype, or Danger}

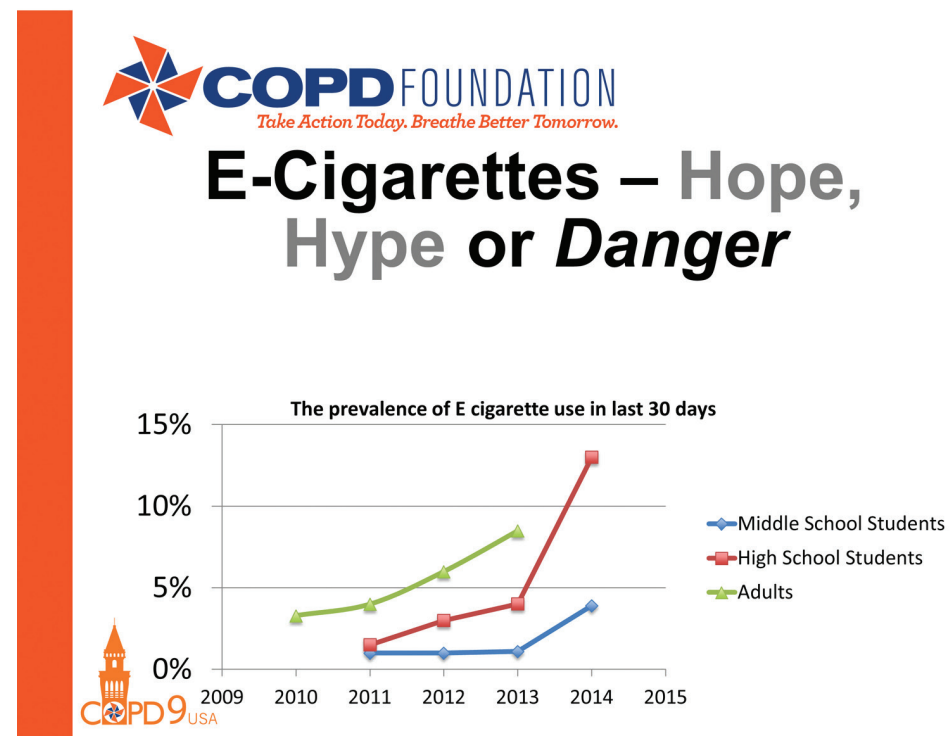

tobacco smoke can also be found in electronic cigarette vapor (albeit in somewhat lower concentrations). ${ }^{7}$ Thus, electronic cigarettes do not currently appear to live up to their promise of being a safer alternative to tobacco products. This is a significant concern given their increased use across age groups.

\section{Looking Forward}

Dr. Prescott Woodruff of the University of CaliforniaSan Francisco looked toward the future with an analysis of how COPD endotypes, (forms of disease based on biological processes not physiological symptoms), rather than phenotypes, may reveal solutions to problems not answered by existing research efforts. Building on Dr. Gerard Turino's discussion of biomarkers, Dr. Woodruff remarked that by understanding the distinct biomolecular processes that are shared by patients with similar biology and similar symptoms, clinicians can theoretically tailor pharmaceutical treatments that target those specific processes, potentially increasing effectiveness and improving outcomes.

In addition to the alpha-1 deficiency endotype previously described by Dr. Turino, Dr. Woodruff posited that additional COPD endotypes may include COPD accompanied by persistent inflammation and what Dr. Woodruff described as high-eosinophilic COPD, which may resemble (or encompass) asthmaCOPD overlap syndrome. These endotypes appear to predispose patients to cycles of infection, inflammation, and airway remodeling/tissue degradation, which lead to worsening symptoms and a decreased quality of life.

According to Dr. Woodruff, understanding the effect a particular endotype of COPD has on pathology and treatment response can have a major impact on a variety of research and treatment areas, including identifying COPD biomarkers, determining appropriate treatment/ management plans, and disease staging. A better understanding of biomarkers and chemical processes indicative of these endotypes may even lead to greater insight into the heterogeneity of COPD symptoms, putting medical science closer to the actual cure encouraged by Dr. Rennard and, in the interim, allowing more efficient and cost-effective treatment for a variety of COPD phenotypes.

\section{The Future is Now: A Patient Perspective}

Perhaps the most unique presentation of the plenary session was the one given by Mr. Jim Nelson of Arizona. 
Mr. Nelson, an accountant by trade, spoke from the patient perspective. Diagnosed with COPD in his 50s after a premature birth and a lifetime of smoking, $\mathrm{Mr}$. Nelson received a double-lung transplant in 2011. With his wife and caregiver Mary at his side, Mr. Nelson has devoted countless hours to COPD advocacy and education (for both fellow patients and clinicians alike) at the state and national levels. The theme of this presentation reminded attendees that for many people living with COPD, the future is, by necessity, now. After a brief biographical summary, Mr. Nelson presented many of the barriers and complications faced by today's COPD patient, from communications breakdowns related to a lack of common vocabulary between clinicians and the populations they serve to issues with resource allocation in the transplant system. In addition, Mr. Nelson spoke to the human cost of COPD exacerbations, notably discussing what he termed the stair-step effect in which a person undergoing an exacerbation will likely never quite regain the same level of lung function as before the flare (as indicated by some of Dr. Rennard's naturalhistory curves ${ }^{8}$ ).

Mr. Nelson's solution to this human cost is for patients and caregivers to take a leading role in COPD management, with the caveat that each must understand the complexities involved in such a responsibility and that this understanding originates with the treatment team. Again, he pointed out that clear and consistent communication is the key to this understanding, and that communication must take place in relatively plain language, rather than medical jargon. As an exemplar, Mr. Nelson quoted the founder of the COPD Foundation, Mr. John Walsh, as crediting his relative increase in functional knowledge to improved communication and understanding.

\section{COPD360 and the Patient-Powered Research Network}

The reference to John Walsh created an ideal segue to the final presentation of the plenary session, given by Mr. Walsh. Mr. Walsh looked briefly to the past, dedicating his presentation to the memory of 2 inspirational figures; Chip Gatchell (one of the founders of the large "COPD International" online support group and an early COPD Foundation board member) and Maggie Borger (one of the initial COPD Foundation state advocacy captains as well as one of the first COPD Information Line associates). In their honor, Mr. Walsh then turned to the future of COPD management, with a description of the COPD Foundation's innovative, patient-centered research and communication initiatives. (Figure 2) COPD360social, the communication initiative was launched in November 2014 as the largest social media platform dedicated specifically to people living with COPD. COPD360social enables stakeholders (patients, clinicians, scientists, et al.) to communicate with each other in a dedicated venue, providing unprecedented access to clinically-relevant information and support.

\section{Figure 2. COPD360 Vision}

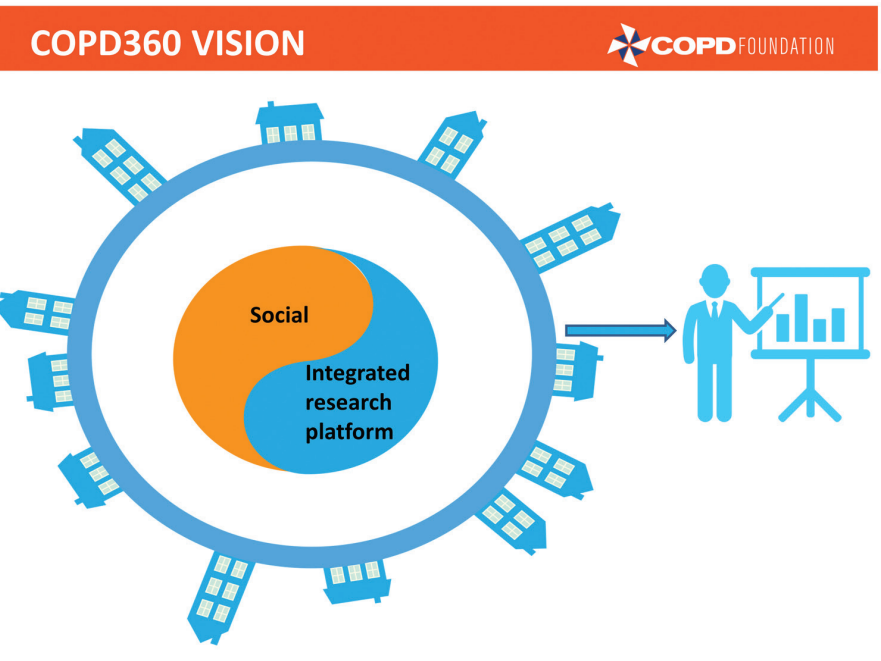

The research companion to COPD360social, known as the COPD Patient-Powered Research Network (COPD PPRN), allows researchers to connect with patient volunteers committed to participating in clinical research initiatives. This connection potentially makes recruitment and data gathering significantly easier, streamlining the research process. It is Mr. Walsh's hope that these 2 components of the COPD360 initiative can support people living with COPD in multiple ways, from education to participation in care improvement. Mr. Walsh has set a goal of 75,000 participants for the PPRN in order to create a viable cohort and, in his words, "change the paradigm of the way clinical research is being done.” Eventually, the PPRN may even interface with electronic medical records for additional clinical information. These initiatives are aimed at tying together all the issues raised by past and present COPD research, including phenotyping (and potentially endotyping), and allow for the creation of the targeted treatment modalities that are needed to address the heterogeneity of COPD cases. Mr. Walsh then concluded with an exhortation to patients and clinicians alike to assist in 
recruitment and development of both COPD360social and the PPRN, and provided a variety of methods both groups can support and access these initiatives.

This plenary session was a representation of the philosophy of COPD360. By taking a look at where research has been, what the current issues are, and what the future may hold, the session offered attendees a rich understanding of the complexities of diagnosis and treatment of COPD. In addition, by including a variety of viewpoints, including the experience of those actually living with the disease, the session provided an opportunity to see multiple insights of COPD. Clinicians have the opportunity to build on what has come before, and take COPD science back to the future.

\section{Declaration of Interest}

Dr. Rennard has received reimbursement for attending a symposium, speaking and/or consulting from: ABIM, Able Associates, Advantage Healthcare, Align2Action, Almirall, APT, ATS, AstraZeneca, Baxter, BoehringerIngelheim, Chiesi, CIPLA, ClearView Healthcare, Cleveland Clinic, CME Incite, Complete Medical Group, COPD Foundation, Cory Paeth, CSA, CSL, CTS Carmel, Dailchi Sankyo, Decision Resources, Dunn Group, Easton Associates, Elevation Pharma, FirstWord, Forest, Frankel Group, Gerson, GlaxoSmithKline, Gilead, Grifols, GroupH, Guidepoint Global, Haymarket, HealthStar, Huron Consulting, Incite, Inthought, IntraMed (Forest), Johnson \& Johnson, LEK, McKinsey,
Medical Knowledge, Mediummune, Methodist Health System (Dallas), Navigant, NCI Consulting, Novartis, Nuvis, Pearl, Penn Technology, Pfizer, PlanningShop, Prescott, Pro Ed Comm, ProiMed, PSL FirstWord, Pulmatrix, Quadrant, Qwessential, Regeneron, Saatchi and Saatchi, Schlesinger Associates, Strategic North, Synapse, Takeda, Theron, WebMD. Dr. Rennard's institution has received grant support from the National Heart Lung and Blood Institute, Nebraska Department of Health and Human Services, Otsuka, Pfizer, GlaxoSmithKline, Boehringer Ingelheim, Nycomed, AstraZeneca, Centocor, Almirall. He is currently an employee of AstraZeneca. Dr. Turino has served as a consultant for Baxter and has received grants from CSL Behring and Boehringer Ingelheim. Dr. Bowler has served as an advisor and received grants from Boehringer Ingelheim. He has also received a grant from MedImmune. Dr. Woodruff has served as an advisor for Janssen, Neostem and Genentech. He has been a consultant for Roche, Novartis and AstraZeneca and has received a grant from Genentech. Dr. Make has participated in research studies and/or served on medical advisory boards for AstraZeneca, BoehringerIngelheim, GlaxoSmithKline, Novartis, Spiration, and Sunovion.

NOTE: To complete the CME post test for this article, refer to the original online version of the article at:

\section{www.journal.copdfoundation.org}




\section{References}

1. Fletcher C, Peto R. The natural history of chronic airflow obstruction. Br Med J. 1977; 1(6077): 1645-1648. doi: http://dx.doi.org/10.1136/bmj.1.6077.1645.

2. Global initiative for chronic Obstructive Pulmonary Disease (GOLD). Documents and Resources. GOLD website. http:// www.goldcopd.org/guidelines-resources.html Published 2015. Accessed December 2015.

3. Massaro GD, Massaro D. Retinoic acid treatment abrogates elastase-induced pulmonary emphysema in rats. Nat Med. 1997; 3(7): 675-677 doi: http://dx.doi.org/10.1038/nm0897-817b.

4. Chapman KR, Burdon JGW, Piitulainen E, et al. Intravenous augmentation treatment and lung density in severe a1 antitrypsin deficiency (RAPID): a randomised, double-blind, placebocontrolled trial. Lancet. 2015; 386(9991): 360-368 doi: http://dx.doi.org/10.1016/S0140-6736(15)60860-1.

5. Bullen $\mathrm{C}$, Howe $\mathrm{C}$, Laugesen $\mathrm{M}$, et al. Electronic cigarettes for smoking cessation: a randomised controlled trial. Lancet. 2013; 382(9995):1629-1637

doi: http://dx.doi.org/10.1016/S0140-6736(13)61842-5 .

6. Caponnetto P. Campagna D, Cibella F, et al. EffiCiency and Safety of an eLectronic cigAreTte (ECLAT) as tobacco cigarettes substitute:A prospective 12-Month randomized control design study. PLOS One. 2013; 8(6): e66317 doi: http://dx.doi.org/10.1371/journal.pone.0066317

7. Goniewicz ML, Kynsak J, Gawron M, et al. Levels of selected carcinogens and toxicants in vapour from electronic cigarettes. Tob Control. 2014; 23:133-139 doi: http://dx.doi.org/10.1136/tobaccocontrol-2012-050859.

8. Rennard SI, Drummond B. Early chronic obstructive pulmonary disease: definition, assessment and prevention. Lancet. 2015; 385:1778-1788.

doi: http://dx.doi.org/10.1016/S0140-6736(15)60647-X 